\title{
Hiperprolactinemia y Esterilidad Conyugal
}

\author{
Dr. Gilberto Angel $\mathbf{M}$
}

\section{Resumen}

La prolactina es producida por el lóbulo anterior de la hipófisis y regulada por el sistema nervioso central e hipotálamo. La hiperprolactinemia bloquea la acción gonadotrófica de las hormonas foliculo estimulante (FSH) y luteinizante (LH) provocando un retardo en la actividad ciclica del ovario, manifestada clinicamente por ciclos irregulares o permanecer asintomática, pero produciéndose invariablemente insuficiencia luteínica, que es evidente histológicamente en los endometrios pre-menstrual o menstrual, con ausencia o deficiencia en la reacción pseudo decidual, indispensable para la implantación del embrión, y de arterias espirales que desempeñan un importante papel en la placentación posterior

La depuración y a la vez popularización de las técnicas de radio-inmunoensayo en Laboratorio Clínico, han permitido verificar más investigaciones hormonales, derivándose de su conjunto valiosos aportes prácticos, pues las dificultades anteriores en las dosifica. ciones hormonales hacian que muchos conceptos permanecieran en el terreno de lo académico sin traspasar al terreno cle la práctica. La prolactina permaneció durante tiempo prolongado, como indicadora de procesos tumorales en la

\footnotetext{
* Médico Patólogo Clinico Jefe Laboratorio Médico Clínica Los Remedios - Cali
}

silla turca y de su actividad lactogénica. En el año de 1971 se reconoció en la prolactina sus acciones bloqueadoras en las hormonas gonadotróficas y es prácticamente descle dicho tiempn que toman auge sus determinaciones y múltiples aplicaciones

Sus niveles elevados se asocian con hipogonadismo tanto masculino como femenino, desarrollando sintomatologias larvadas, evidentes, o simplemente consecuentes como sucede en la mujer infértil asintomática, donde la prolactina está ejerciendo su función fisiológica anticonceptiva, sin producir lactancia.

\section{Histofisiologia}

El lóbulo anterior de la hipófisis, produce unas 20 sustancias y entre ellas la prolactina, producida por las células lactotrópicas. Su secreción está regulada por el sistema nervioso central y el hipotálamo, al ponerse éste en relación directa con su producción, por medio de capilares tipo porta localizados en el tercer ventrículo en su eminencia media. Grupos especializados de células hipotalámicas de ésta región, segregan una serie de compuestos denominados 'factores hipotalámicos' que estimulan o inhiben la secreción de hormonas hipofisiarias. Tiene la prolactina la particularidad especial que mientras las otras hormonas antero-hipofisiarias descienden sus niveles con estímulo hipotalámico, la prolactina los aumenta, lo que explica que lesiones tumorales de la silla turca evolucionen con au- 
mento de la prolactina y descenso de las otras hormonas. (15-17-19).

El factor hipotalámico que inhibe la producción de prolactina, se ejerce a través de un mecanismo intervenido por catecolaminas y representado por la DOPAMINA (20-24-25) encontrándose en ésta sustancia el principal factor inhibitorio conociéndose con el nombre de PIF (prolactin inhibiting factor). Recientes investigaciones han demostrado ampliamente que el alcaloide sintético ergot, la BROMOCRIPTINA ejerce las mismas acciones inhibitorias administrándolo por via oral, y ha sido quizás está comprobación, la que ha suscitado numerosas publicaciones relacionadas con la prolactina, al desaparecer sindromes ocasionados por hiperprolactinemia con el uso del alcaloide.

\section{Fisiologia de la prolactina}

Es una proteína que está integrada por cerca de 190 aminoácidos (21) y hoy en dia por técnicas radio-inmunológicas se pueden obtener en una glándula hipofisiaria cerca de 100 microgramos. Su estructura quimica es muy similar a la de las hormonas somatotrópicas. Los factores de liberación conocidos como PRF (prolactin releasing factor) (22) estimulan normalmente la lactancia. Cuando ésta se ejerce, simultáneamente se bloquea la acción gonadotrófica de las hormonas $\mathrm{LH}$ y FSH provocando un retardo en la actividad cíclica del ovario, manifestada en este caso por acción anticonceptiva natural y fisiológica, que en la práctica se traduce en esterilidad transitoria de la mujer lactante. Pero cuando sus niveles aumentan no a expensas de factores de liberación (PRF) sino por variaciones en los niveles inhibitorios (PIF), se obtienen fisiológicamente los mismos resultados que en la mujer lactante, con la consecuente infertilidad que muchas veces pasa totalmente asintomática, pero ejercida por la prolactina por su acción anticonceptiva.
La concentración en la sangre varía según la técnica empleada y la calidad del suero inmune empleado. Si se expresa un $\mathrm{mU} / \mathrm{L}$ corresponde a cifras entre 30 y $360 \mathrm{mU} / \mathrm{L}$ y si se expresa en unidades masa la normalidad se encuentra entre 1.5 y $18 \mathrm{ng} / \mathrm{L}$. Sus niveles se elevan con el sueño y vigilia, por lo que se prefiere tomar la muestra para su dosificación entre las 9 a.m. y 4 p.m. (no se requiere ayuno previo).

\section{Prolactina y esterilidad}

Siempre se debe tener presente el nivel de prolactina en toda mujer infértil como estudio complementario, después de descartar el factor masculino que a veces se olvida desempeña un papel del $50 \%$. Normalmente las hormonas FSH y $\mathrm{LH}$ son liberadas en el lóbulo anterior, estimulando la función ovárica en sus fases folicular y luteínica, verificándose la ovulación, e interviniendo el cuerpo amarillo en la maduración del endometrio, acondicionándolo para recibir el óvulo fecundado.

Normalmente está presente en la capa compacta después del $24^{\circ}$ día del ciclo (34-36) reacción pseudo-decidual rica en glucógeno, cuya función primordial es la nutrición del embrión en los primeros momentos de la implantación evitando así que "muera de hambre". (34). Su acción, se ejerce igualmente sobre las arterias espirales, que al final del ciclo son histológicamente fácilmente reconocibles en la capa superfi. cial y esponjosa y van a tener importancia capital en la placentación posterior. El aumento de la prolactina (frecuente después de la supresión de anticonceptivos) (30) o cualquier causa que la eleve, bloquea la acción de la $\mathrm{LH}$, se pro. duce insuficiencia luteínica y ésta se manifiesta histológicamente en el endometrio, bien sea en maduración irregular de las glándulas o lo que es más frecuente en alteraciones del estroma con ausencia de reacción pseudo-decidual y arterias espirales. A cambio de ésto, predomina el edema intersticial lo 
que constituye en su conjunto, un endometrio totalmente inapto para la implantación o siembra, porque el terreno no tiene el abono suficiente e incapaz de proporcionar una placentación eficiente por falta de riego proporcionado por las arterias espirales, todo por una insuficiencia luteínica, motivada por exceso de prolactina y ejercida sobre un terreno donde se pretende verificar una siembra. $(26,28,30,34,35$ y 36).

\section{Bibliografía}

Trabajos presentados en el IX Congreso Mundial de Ginecología y Obstetricia, octubre 25 al 31, 1979 - Tokio. Abstracts publicado por la FIGO, Tokio, Obre, 1979.

1 No. 217: Pág. 97: A study of galactorrhoea, etiology and management. Kanjilal and Cols. College-Hospital Calcutta-India.

2 No. 218: Pág. 98: Failure of pyrodoxine B6) o inhibit serotonin stimulated prolactine release in non lactating women. Foukas and Cols. Medical Schhol-AthenasGRIECE.

3 No. 219: Pág. 98: Plasma prolactin responses to thyrotrophin releasing hormone in momen with idiopathic hyperprolactinemia. Evans and Cols. Hospital London-UNITED KINGDOM.

4 No. 220: Pág. 99: Clinical course and outcome of pregnancy in 25 patients with pituitary microadenomas.

Jawlewicz and Col. College of Phys. Surg. NEW YORK.

5 No. 230: Pág. 103: Reversal of the day-night rhytm of serum prolactin in policystic ovarian.

Gorrafa -Aly School of Medicine- Dayton (Ohio) U.S.A.

6 No. 231: Pág. 104: Estrogen dependence of prolactin secretion in the policystic ovary sindrome. Results of bromocriptine treatment.

Del Pozo y Colab. University of Rome-ITALIA.

7 No. 236: Pág. 106: Effect of lisurid (Ergot derivate) in the treatment of hyperprolactinemia and galactorrhoea.

Tempone A y Colab. Cátedra Ginecología -BUENOS AIRES-
8 No. 237: Pág. 106: The effect of lisuride in the hyperprolactinemia, amenorrhoea sydrome and puerperal lactation.

De Cecco L-Clínica Ostetricia -GenoveITALIA.

9 No. 238: Pág. 107: The efect of lisuride in hiperprolactinemia states.

Floersheim and Cols. Dept. Gynec. Endo. Zirich-SWITZERLAND.

10 No. 239: Pág. 107: The treatment with 2 brom alfa ergocriptin (Parlodel) in the syndrome amenorrhoea galactorreaoe.

Sirbu and Cols. Depto. Obst. and Gynec. Bucharest-RUMANIA.

11 No. 254: Pág. 112: Prolactin levels in postmenopausal women.

Castellanos y Colab. Clin. San Jordi-BarceIona-ESPAÑA.

12 No. 279: Pág. 126: Therapeutic effect of bromocryptine in anovulatory women with normal prolactin levels.

Waronski W. Acad. Inst. Obst. and Gynec. Katowicwe-POLAND.

13 No. 293: Pág. 133: Ergocryptine treatment in high prolactin sterility.

Borenstein and Col. Univ. Med. School. Rehovot-ISRAEL.

14 No. 299: Pág. 136: The course and outcome of pregnancy after ovulation induction with short term bromocryptine therapy. Klimek R. Inst. Obst. and Gynec. GracowPOLAND.

15 No. 301: Pág. 137: Determination of ketoprostaglandin, $\mathrm{LH}, \mathrm{FSH}$ and prolactin in seminal fluid of fertile and infertile men.

Klinge K. Runnebaunm. B. Univ. Frauneklinik-HEIDELBER.

16 No. 177: Pág. 79: Modulation of the regulatory mechanisme of prolactin secretion. S. Scapagnini U. and Colbs. Univ. of CataniaITALIA.

17 No. 179: Pág. 80: Evaluation of pituitary function tests in hyperprolactinemic amenorrhoea.

Shaw R-Glass M-Dept. Obst. and Ginec Birmingham-UNITED KINGOM.

18 No. 182: Pág. 81: Sex diference in the hormonal regulation of human prolactin receptor in rast livers.

Furuhashi N - Tohoku Univ. Myyagi- JAPAN.

19 No. 183: Pág. 82: Biosynthesis and rekease of prolactin.

Hoshino K-Kyoto Univ. Kyoto-Tokio-JAPAN. 
20 Crosignani P. G. and Robyn C.

Prolactin and human reproduction -Crosignani and Robyn- Academic. Press. New York, 1977.

21 Human pituitary pro!actin-Shome $B$ and Parlow A.

J. Clin. Endocrin: 45: 1112, 1977.

22 Galactorrehoea-Treatment with normocryp tine-Besser G. M.

J. Med. 1669, 1972.

23 Hyperprolactinemic anovulatory syndrome. Bohnet H. G.

J. Clin. Endocrin. Metab. 42: 132, 1976.

24 Studies of mechanism of the dopamine mediate inhibition of prolactin secretion. Macleod R. and Lehmeyer.

J. Endocrin. 94: 1077, 1974

25 Effect of ergot drugs on central cathecholamine neurona. Corrodi H. Fuke - J. Pharm. Pharmacol. 25: 409, 1973.

26 Incidence and significance of hyperprolactinemia in women with amenorrhoea Kranks S-Murray $M$

Clin Endocrin. 4: 597, 1975.

27 Prolactin and gonadotrhophin interaction. Thorner M. O. - Edwards C- Press New York, 1970 .
28 Bromocriptine therapy of female infertility. Thorner M. O. Brit. Med. J. 4: 694, 1975

29 An abnormality of prestrogen feed back in amenorrhoea. Glass M. R. -Shaw R. W-. Med. J. End 3: 274. 1976

30 Sterility and considerations. Bohnet M. G J. Clin Endocrin. 42: 132. 1976

31 What, Why and Weere prolactin? E. Del Pozo Sandorama II/1979. Sandoz Ltda.

32 Tratamiento del hipogonadismo con bromocriptina. -Thorner M. O and Besser-. Simposio del Colegio Real de Médicos. Pág. 54-62. Londres, 1976.

33 Evaluación clinica por el Laboratorio Gilberto Angel M. - Edit. Tercer MundoBogotá, marzo 1979

34 Patologia y fisiologia del endometrio-Sammartino R. Simposium para GraduadosMendoza (Argentina). Julio 6, 13, 1959.

35 ESTIRILIDAD-Tyler T. E. Edit. Méd. Panamericana, Buenos Aires. 1973.

36 Esterilidad conyugal. Ahumada J. C. y Zolaboradores -Edit. Panamericana- Buenos Aires, 1964 (Cap. endometrio) 